\title{
Exchange Rate Constants of Invisible Protons in Proteins Determined by NMR Spectroscopy
}

\author{
Takuya Segawa, ${ }^{[a]}$ Fatiha Kateb, ${ }^{[b, d]}$ Luminita Duma, $^{[b]}$ Geoffrey Bodenhausen, ${ }^{[b, c]}$ and \\ Philippe Pelupessy*[b]
}

\begin{abstract}
Although labile protons that are exchanging rapidly with those of the solvent cannot be observed directly, their exchange rate constants can be determined by indirect detection of scalar-coupled neighboring nuclei. We have used heteronuclear NMR spectroscopy to measure the exchange rate constants of labile protons in the side chains of lysine and arginine residues in ubiquitin
\end{abstract}

enriched in carbon-13 and nitrogen-15 at neutral pH. Exchange rate constants as fast as $40 \times 10^{3} \mathrm{~s}^{-1}$ were thus measured. These results demonstrate that NMR spectroscopy is a powerful tool for the characterization of lysine $\mathrm{NH}_{3}{ }^{+}$and arginine $\mathrm{NH}$ groups in proteins at physiologically relevant $\mathrm{pH}$ values.

\section{Introduction}

Proton-exchange rate constants provide a rich source of structural and dynamic information. ${ }^{[1-3]}$ These rate constants can be used to study important kinetic processes such as the opening of base-pairs in nucleic acids ${ }^{[4,5]}$ and give insight into free energies and other thermodynamic parameters that describe the stability of hydrogen-bonded secondary structures under different environmental conditions. ${ }^{[6]}$ NMR is often the method of choice to measure proton-exchange rates at individual sites in complex systems. ${ }^{[7-10]}$ By comparing proton-exchange rate constants measured in proteins with those observed in model systems, one can determine so-called protection factors and identify residual structures in partly denatured proteins. ${ }^{[11,12]}$ Chemical exchange dependent saturation transfer $(C E S T)^{[13-16]}$ and water relaxation $^{[17]}$ can be used to enhance image contrast in MRI. This allows one to assess pathological disorders that cause a modification of water exchange rates.

Most proton-exchange studies in proteins reported so far have been restricted to backbone amide protons. The exchange rate constants of labile protons in side chains have rarely been studied, although these surface-exposed protons often play crucial roles in inter- and intramolecular interactions. The appearance or disappearance of signals corresponding to side chain protons can thus be regarded as an indication of the formation or disruption of protein complexes. ${ }^{[18]}$

For a small molecule containing a single exchanging proton, the maximum rate constant that can be determined by detection of ${ }^{1} \mathrm{H}$ signals is a few thousand jumps per second, if the chemical shift of the exchanging proton is separated from the resonance of the solvent by a few $\mathrm{kHz}$. If there are several exchanging sites, or if the proton spectrum is crowded, correlations with ${ }^{15} \mathrm{~N}$ spectra may be needed to resolve the resonances. ${ }^{[19,20]}$ However, the efficiency of coherence transfer from labile protons to neighboring ${ }^{15} \mathrm{~N}$ nuclei $\left[J\left({ }^{1} \mathrm{H}^{15} \mathrm{~N}\right) \approx 100 \mathrm{~Hz}\right]$ is severely hampered when the exchange rate constants are comparable to the scalar coupling constants, that is, if $k_{\text {ex }}$ exceeds about $100 \mathrm{~s}^{-1}$.
For intermediate exchange rate constants $k_{\mathrm{ex}} \approx \pi J\left({ }^{1} \mathrm{H}_{1}^{15} \mathrm{~N}\right)$, the doublets in the ${ }^{15} \mathrm{~N}$ spectra collapse to broad lines, which become narrower when the exchange rate constants exceed the scalar coupling constants. If this narrowing effect is incomplete, the remaining linewidth gives a measure of the exchange rate constants $k_{\text {ex }}$ ("scalar relaxation of the second kind"). ${ }^{[21]}$ The residual widths of the collapsed lines in the ${ }^{15} \mathrm{~N}$ spectra can be reduced by proton decoupling, so that the comparison of proton-coupled and -decoupled ${ }^{15} \mathrm{~N}$ spectra gives a measure of the exchange rate constants of the attached protons. ${ }^{[22,23]}$ However, this method is not very accurate when the ${ }^{15} \mathrm{~N}$ spectra are affected by long-range couplings ${ }^{n} \mathrm{~J}$ $\left({ }^{1} \mathrm{H}^{15} \mathrm{~N}\right)$ with $n>1$. Recently, we have introduced a method to quantify the effects of scalar relaxation caused by exchanging protons, by detecting the decay of a ${ }^{15} \mathrm{~N}$ coherence under a multiple-refocusing Carr-Purcell-Meiboom-Gill (CPMG) pulse $\operatorname{train}^{[24,25]}$ in the presence or absence of proton decoupling. ${ }^{[2]}$ Refocusing removes the effects of long-range couplings even in the absence of proton decoupling. For the indole proton in tryptophan, we were able to detect exchange rate constants $k_{\text {ex }}$ accurately up to $10^{5} \mathrm{~s}^{-1} \cdot{ }^{[26]}$ In this work, this idea is extended to the indirect detection of labile $\mathrm{H}^{\mathrm{N}}$ protons in the side

[a] T. Segawa

Department of Chemistry, Eidgenössische Technische Hochschule 8093 Zürich (Switzerland)

[b] Dr. F. Kateb, Dr. L. Duma, Prof. G. Bodenhausen, Dr. P. Pelupessy École Normale Supérieure

24 rue Lhomond, 75231 Paris (France)

Fax: $(+33)$ 1-44-32-33-97

E-mail:Philippe.Pelupessy@ens.fr

[c] Prof. G. Bodenhausen

École Polythechnique Fédérale de Lausanne

Laboratoire de Résonance Magnétique Biomoléculaire, Batochime 1015 Lausanne (Switzerland)

[d] Dr. F. Kateb

Present address: Institute of Structural Biology

Ingolstädter Landstrasse 1, 85764 Neuherberg (Germany) 
chains of lysine and arginine residues in proteins. This method is not limited to the arginine and lysine side chains and can be extended to the measurement of the exchange rate constants of the backbone and side chain protons of other amino acids in proteins as well as imino protons in DNA or RNA.

\section{Results and Discussion}

\section{Theory}

We consider an isolated spin pair IS, in which spin I (usually a proton ${ }^{1} \mathrm{H}$ ) exchanges with the solvent, which is typically an aqueous buffer or plain water, while $S$ (usually ${ }^{15} \mathrm{~N}$ ) is chosen because it features a scalar coupling $J_{\text {IS }}$. If a radio-frequency field is applied on-resonance to the I nucleus, the evolution of the $S_{y}$ coherence at the time of the echoes in a multiple-refocusing CPMG pulse-train can be described in a base of only three product operators $\left\{S_{y}, 2 S_{x} I_{z}, 2 S_{x} I_{y}\right\}$. For the $n$th echo, one obtains the following density operator [Eq. (1)]:

$\rho(t=2 n \tau)=\left[\exp (-L \tau) \cdot R_{\mathrm{N}} \cdot \exp (-L \tau)\right]^{n} \rho(0)$

where $R_{\mathrm{N}}$ describes a $\pi_{\mathrm{y}}$ pulse applied to the $\mathrm{S}\left({ }^{15} \mathrm{~N}\right)$ nuclei [Eq. (2)]:

$R_{N}=\left(\begin{array}{ccc}1 & 0 & 0 \\ 0 & -1 & 0 \\ 0 & 0 & -1\end{array}\right)$

and $L$ is the Liouvillian [Eq. (3)]:

$$
L=\left(\begin{array}{ccc}
R_{2}^{S} & \pi J_{I S} & 0 \\
-\pi J_{I S} & R_{2}^{S}+k & -\omega_{1}^{1} \\
0 & \omega_{1}^{\prime} & R_{2}^{S}+k^{\prime}
\end{array}\right)
$$

Here, $\omega_{1}^{1}$ is the amplitude of the decoupling field applied to the I ( $\left.{ }^{1} \mathrm{H}\right)$ nuclei, $R_{2}^{\mathrm{S}}$ is the transverse relaxation rate constant of the $\mathrm{S}\left({ }^{15} \mathrm{~N}\right)$ nuclei, and $k \approx k_{\mathrm{ex}}+R_{1}^{1}$ and $k^{\prime} \approx k_{\mathrm{ex}}+R_{2}^{1}$. If the decoupler is applied off-resonance, the Liouvillian must be expanded to a four-dimensional space $\left\{S_{y}, 2 S_{x} I_{z}, 2 S_{x} I_{y}, 2 S_{x} I_{x}\right\}$. The ratio between the signal intensities in experiments without $(A)$ and with $(B)$ proton decoupling is given by:

$(A / B)_{m=1}=\left\langle S_{y}\right\rangle_{A}\left(t=2 n \tau ; \omega_{1}^{1}=0\right) /\left\langle S_{y}\right\rangle_{B}\left(t=2 n \tau ; \omega_{1}^{1} \neq 0\right)$

The case of $m=1$ corresponds to a single exchanging proton in a $-\mathrm{NH}$ spin system. This ratio does not depend on $R_{2}^{S}$ because it equally affects the signal intensities in both experiments. For $m$ chemically equivalent protons $(m=2$ or 3 describe $-\mathrm{NH}_{2}$ or $-\mathrm{NH}_{3}{ }^{+}$systems), it can be shown that:

$(A / B)_{m=3^{1 / 3}}^{1 / 3}=(A / B)_{m=2}^{1 / 2}=(A / B)_{m=1}$

Thus, the effect of proton exchange on the decay of the signal intensity of the $S$ nuclei is enhanced with an increasing number of protons. Provided that $\omega_{1}^{1} \gg R_{2}^{1}$, the ratios in Equations (4) and (5) do not depend on $R_{2}^{1}$. Hence, if the scalar coupling is known, the only unknown parameter in these equations is $k$. For the exchange rate constants measured in this work, $k \approx k_{\text {ex }}$. When $k_{\text {ex }}$ is small, an independent estimate of $\mathrm{R}_{1}^{1}$ is needed for an accurate determination of $k_{\text {ex }}$.

\section{Methodology}

The pulse sequences designed to detect the exchange rate constants of the labile $\mathrm{H}^{\mathrm{N} \varepsilon}$ protons of arginine and the three terminal $\mathrm{H}^{\mathrm{N} \zeta}$ protons of lysine side chains are drawn in Figure 1.

The polarization of the neighboring $\mathrm{H}^{\delta}$ (for arginines) or $\mathrm{H}^{\varepsilon}$ (for lysines) is converted into antiphase coherences $2 N_{y}^{\varepsilon} C_{z}^{\delta}$ and $2 N_{y}^{\zeta} C_{z}^{\varepsilon}$ in arginine and lysine, respectively, through two successive coherence transfer steps in the manner of Insensitive Nuclei Enhanced by Polarization Transfer (INEPT). ${ }^{[32]}$ These antiphase coherences are then allowed to evolve under a multiple-refocusing CMPG pulse train, with or without proton decoupling (experiments $B$ and $A$ ). For the arginine residues, the antiphase coherence $2 N^{\varepsilon}{ }_{y} C_{z}$ is transferred back to the neighboring $\mathrm{H}^{\delta}$ protons, after a constant-time evolution under the chemical shift of $C^{\delta}$. For the lysine side chains, the coherence $2 N_{y}^{\zeta} C_{z}^{\varepsilon}$ is transferred to $C^{\delta}$ via $C^{\varepsilon}$ and finally detected on the remote $\mathrm{H}^{\delta}$ protons, after a constant-time evolution under the chemical shift of $C^{\delta}$. An additional transfer step from $C^{\varepsilon}$ to $C^{\delta}$ is necessary in ubiquitin because the $\mathrm{H}^{\varepsilon} / \mathrm{C}^{\varepsilon}$ correlation signals of the seven lysine residues are ill-resolved.

\section{Discussion}

Under physiological conditions, lysine $\mathrm{NH}_{3}$ and arginine $\mathrm{NH}$ side chain groups are positively charged. Lysines and arginines are often found in protein binding sites and, through the formation of salt bridges with carboxylate groups of glutamate and aspartate or phosphate groups of DNA and RNA, they play key roles in protein-protein interactions and protein-nucleic acid recognition. We have measured the exchange rate constants of $\mathrm{H}^{\mathrm{N} \varepsilon}$ protons in arginine and of the three terminal $\mathrm{H}^{\mathrm{N \zeta}}$ protons in lysine side chains of human ubiquitin at $\mathrm{pH} 7.45$ as a function of temperature.

Representative spectra obtained with the experiments $A$ and $B$ of Figure 1 are shown in Figure 2. The spectra recorded with experiment $B$, where the protons are decoupled during the ${ }^{15} \mathrm{~N}$ refocusing period, are plotted on the right-hand side of Figure 2.

The peaks in these spectra are more intense than those of experiment $A$, shown on the left, which were obtained without proton decoupling, since exchange-induced decay ("scalar relaxation") is only effective in case A. All the arginine and six of the seven lysine cross peaks could be identified (the intensity of K27 was too weak to be exploited).

The exchange rate constants thus measured are given in Table 1 and are plotted on a logarithmic scale against the inverse temperature in Figure 3 for arginines and in Figure 4 for lysines. The linear fits indicate that the exchange constants obey the Arrhenius equation:

$k_{\mathrm{ex}}=A \exp \left(-E_{\mathrm{a}} / R T\right)$ 
A)

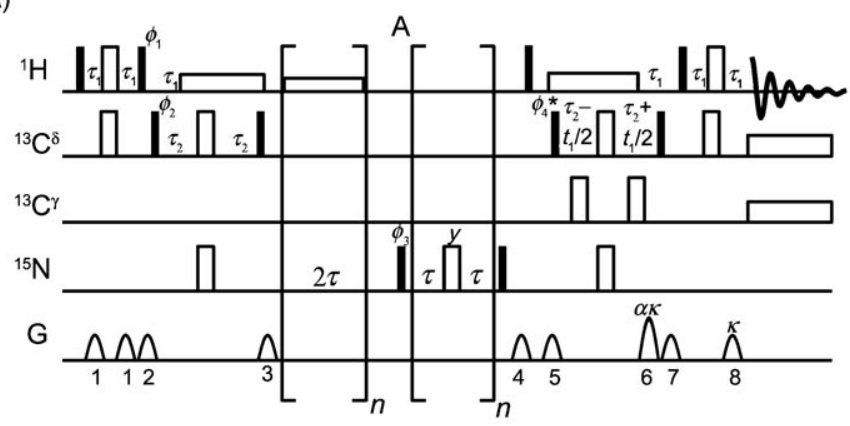

B

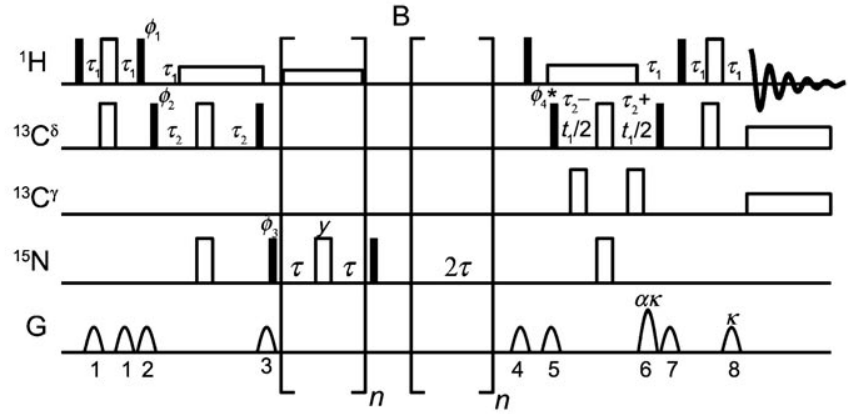

B)

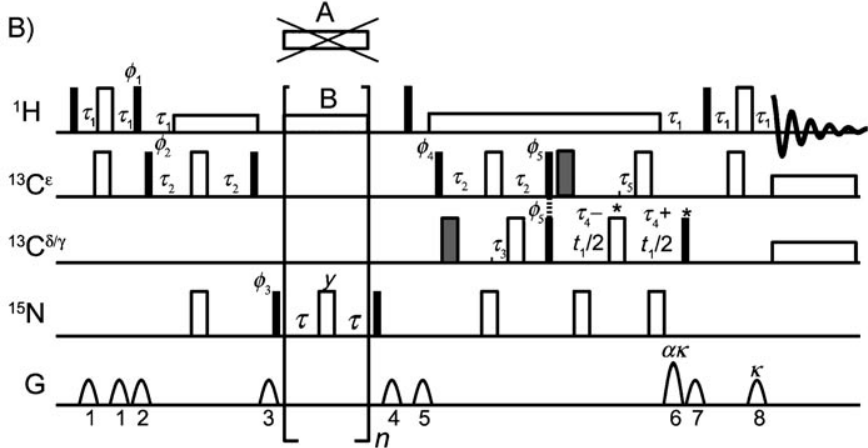

Figure 1. Pulse sequences designed to measure hydrogen exchange rate constants $A$ ) of the $H^{\mathrm{N} \varepsilon}$ protons in arginine, and B) of the three terminal $\mathrm{H}^{\mathrm{N} \zeta}$ protons in lysine residues. Narrow open and filled rectangles indicate $\pi$ and $\pi / 2$ pulses, respectively, while the wide rectangles represent decoupling sequences. For lysine residues, decoupling is applied only in experiment $B$. For arginine residues, proton decoupling is applied in both experiments $A$ and $B$, but the refocusing pulses are delayed until after this decoupling interval in experiment $A$, while in experiment $B$ they are applied at the same time as proton decoupling. This allows one to remove zero-quantum artifacts due to operators such as $8 \mathrm{~N}^{\varepsilon}{ }_{y} \mathrm{C}_{z}{ }_{2} H^{\delta 1}{ }_{y} H^{\delta 2}{ }_{y}$ that contain two-spin coherence terms $2 H^{\delta 1}{ }_{y} H^{\delta 2}{ }_{y}$ which otherwise would evolve differently in experiments $\mathrm{A}$ and $\mathrm{B}$. For lysine residues, this precaution was not needed, since only a negligible part of two-spin terms such as $2 H^{\varepsilon 1}{ }_{y} H^{\varepsilon 2} y$ is transferred to the observed $\mathrm{H}^{\delta}$ coherence. All $\pi / 2$ and the $\pi$ pulses in the first and last INEPT blocks applied to ${ }^{13} \mathrm{C}$ were nonselective rectangular pulses, while the ${ }^{13} \mathrm{C}$ refocusing pulses in the other INEPT blocks had REBURP profiles ${ }^{[27]}$ with a duration of $2 \mathrm{~ms}$. The other ${ }^{13} \mathrm{C} \pi$ pulses had Q3 profiles ${ }^{[28]}$ with a duration of $1.5 \mathrm{~ms}$. Gray $\pi$ pulses compensate for Bloch-Siegert phase shifts. ${ }^{[29]}$ Proton decoupling was performed by using a WALTZ-16 sequence. ${ }^{[30]}$ The delays were set to $\tau_{1} \approx 1 /\left(4 J_{\mathrm{CH}}\right), \tau_{2} \approx 1 /\left(4 J_{\mathrm{NC}}\right), \tau_{3} \approx 1 /$ $\left(4 J_{\mathrm{NC}}\right)-1 /\left(4 J_{\mathrm{CC}}\right), \tau_{4} \approx 1 /\left(2 J_{\mathrm{CC}}\right), \tau_{5} \approx 1 /\left(4 J_{\mathrm{CC}}\right)$. All phases were along the $x$-axes unless indicated otherwise. The phases were cycled according to $\phi_{1}=8\{y\}, 8\{-y\}, \phi_{2}=2\{x\}, 2\{-x\}, \phi_{3}=x,-x, \phi_{4}=4\{x\}, 4\{-x\}, \phi_{5}=16\{x\}, 16\{-x\}$ with a receiver phase $\phi_{\mathrm{rec}}=\{x,-x,-x, x,-x, x, x,-x,-x, x, x,-x, x,-x,-x, x\}$. Evolution of ${ }^{13} \mathrm{C}^{\delta}$ coherence in the $t_{1}$ intervals occurs in a constant-time fashion, while frequency discrimination is achieved in an echo/antiecho manner ${ }^{[31]}$ by inverting the phases of the pulses marked by asterisks, and by inverting, for each $t_{1}$ increment, the sign of the gradient $\mathrm{G}_{6}$ with a relative amplitude $\alpha=\gamma_{\mathrm{H}} / \gamma_{\mathrm{C}}$. The constant-time evolution is achieved by shifting the ${ }^{13} \mathrm{C}^{\delta}$ refocusing pulses to the left in steps of $t_{1} / 2$. For arginine residues, the ${ }^{15} \mathrm{~N}$ refocusing pulse is also moved to the left in steps of $t_{1} / 2$, while the ${ }^{13} \mathrm{C}^{\gamma}$ decoupling pulses are moved to the left in steps of $t_{1} / 4$. For the lysine experiments, the ${ }^{15} \mathrm{~N}$ and ${ }^{13} \mathrm{C}^{8} \pi$ pulses are moved to the left in steps of $t_{1} / 4$ and $t_{1} / 2$, respectively.

The pre-exponential factors $(A)$ and activation energies $\left(E_{\mathrm{a}}\right)$ are given in Table 2.
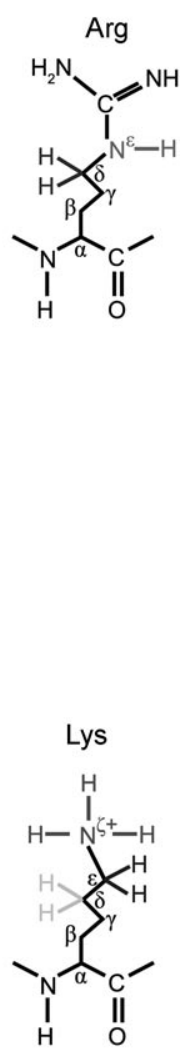

In Figure 3 the exchange rate constants of the $\mathrm{H}^{\mathrm{N} \varepsilon}$ proton in acetylarginine extrapolated from the measurements by Liepinsh and Otting ${ }^{[33]}$ at lower $\mathrm{pH}$ are also plotted as a function of temperature (marked by crosses). The exchange rate constants of residues $\mathrm{R} 42, \mathrm{R} 72$, and R74 are close to these values, while for R54 the rate constants are approximately three times lower. As shown in Figure 5, this is probably due to the fact that the $\mathrm{H}^{\mathrm{N} \varepsilon}$ proton of R54 is less exposed to the solvent and/or because this residue is embedded in a negatively charged environment, which decreases the efficiency of the predominantly $\mathrm{OH}^{-}$-catalyzed exchange mechanism. For the exchange rate constant of R54 at $275 \mathrm{~K}$, the approximation $k \approx k_{\text {ex }}$ might not be valid. The same measurement for a sample of ubiquitin at $\mathrm{pH} 4.5$ resulted in $k=3.1 \pm$ $0.7 \mathrm{~s}^{-1}$, which is an upper value for the contribution of $R_{1}^{l}$.

The exchange rate constant of the $\mathrm{H}^{\mathrm{N \zeta}}$ protons of $\mathrm{K} 6$ is much faster than the others. This residue is very exposed to the solvent, and the neighboring residues are positively charged. The other residues are either less exposed or are embedded in a less positively charged environment. The lowest exchange rate constant is measured for K29, for which evidence exists that ion pairing may occur with D21. ${ }^{[36]}$ The intrinsic exchange rate constant of these protons, as can be extrapolated from the measurements of Liepinsh and Otting ${ }^{[33]}$ in acetyl-lysine at $305 \mathrm{~K}$ and $\mathrm{pH} 7.45$, should be about $7000 \mathrm{~s}^{-1}$. The fact that the exchange rate constants in Table 1 are much higher at this temperature is most probably due to the phosphate buffer, which has been shown to catalyze the exchange of the $\mathrm{H}^{\mathrm{N \zeta}}$ protons in lysine (but not the exchange of the $\mathrm{H}^{\mathrm{N} \varepsilon}$ protons in arginine). Indeed, the addition of $20 \mathrm{~mm}$ phosphate buffer leads to an in- 

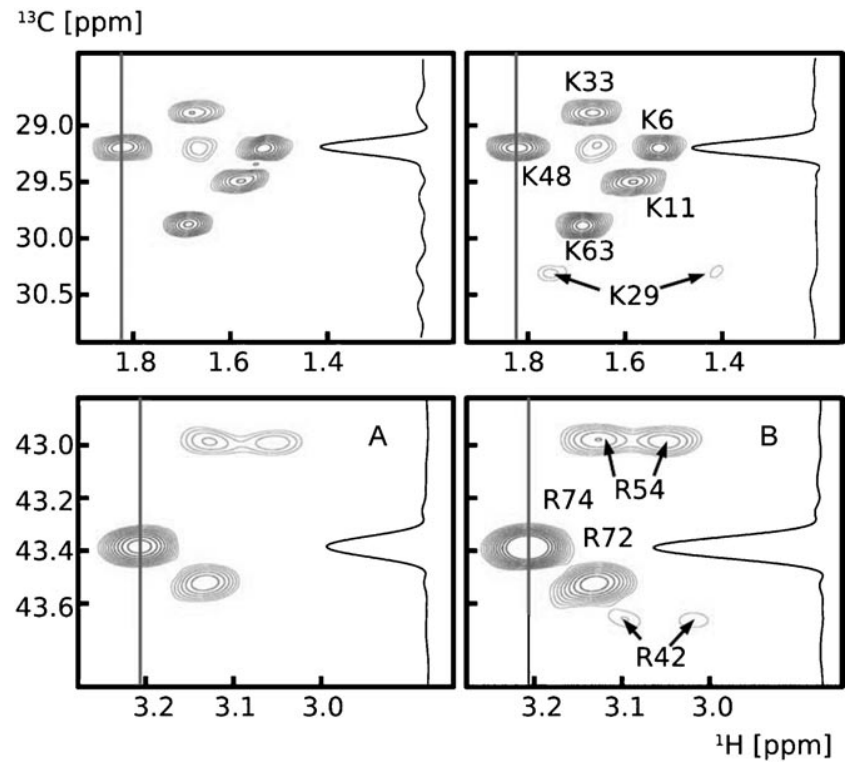

Figure 2. The $\mathrm{C}^{\delta} / \mathrm{H}^{\delta}$ regions of spectra of human ubiquitin at $\mathrm{pH} 7.45$ and $300 \mathrm{~K}$, obtained with the pulse sequences (left panels: experiment $\mathrm{A}$, right panels: experiment $B$ ) of Figure 1 with a number of ${ }^{15} \mathrm{~N}$ refocusing pulses $n=2$ and pulse intervals $2 \tau=10.6 \mathrm{~ms}$ for lysine and $n=4$ and $2 \tau=5.3 \mathrm{~ms}$ for arginine.

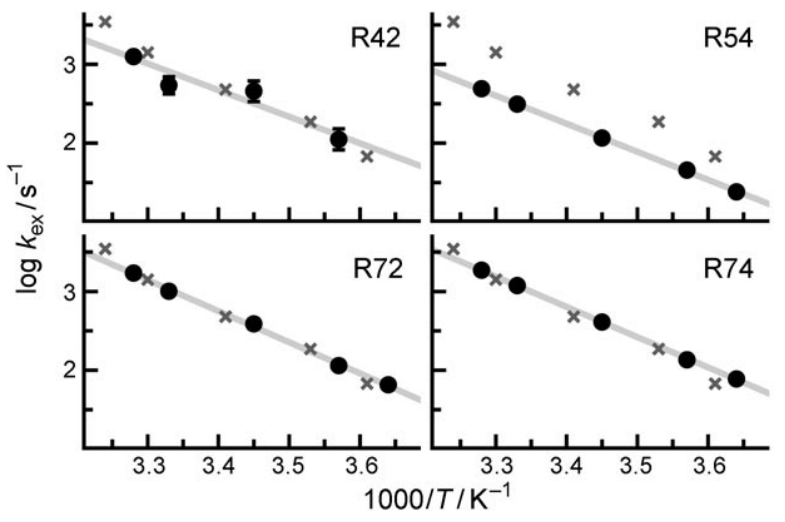

Figure 3. Temperature dependence of the exchange rate constants of the $\mathrm{H}^{\mathrm{N \varepsilon}}$ protons of the four arginine residues in human ubiquitin at $\mathrm{pH}$ 7.45. The linear fits gave the Arrhenius parameters in Table 2. The crosses correspond to an extrapolation to $\mathrm{pH} 7.45$ of the $\mathrm{H}^{\mathrm{N \varepsilon}}$ exchange rate constant obtained by Liepinsh and Otting ${ }^{[33]}$ in acetylarginine at lower $\mathrm{pH}$ values.

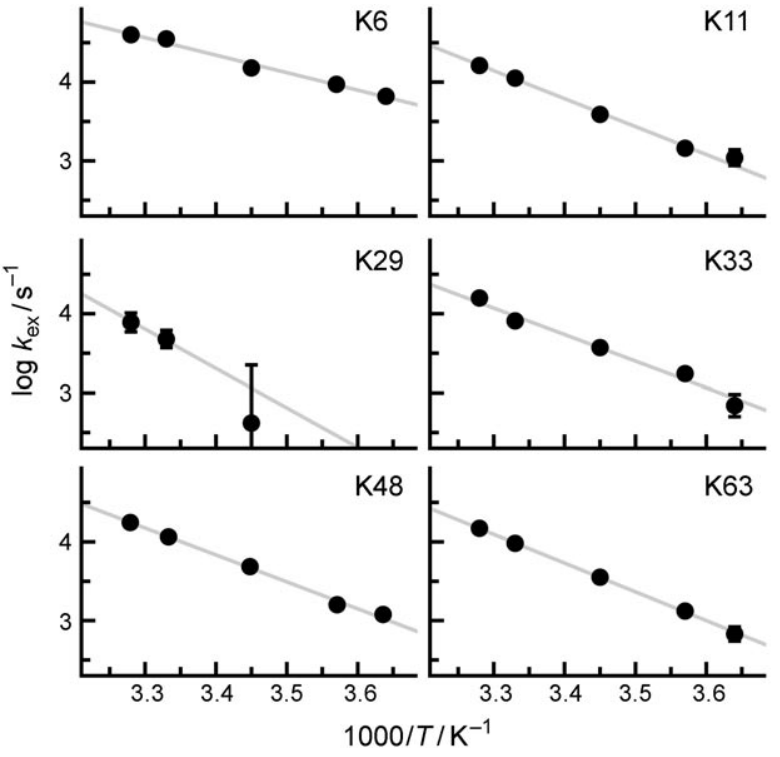

Figure 4. Temperature dependence of the exchange rate constants of the $\mathrm{H}^{\mathrm{N \zeta}}$ protons of six of the seven lysine residues in human ubiquitin at pH 7.45. The linear fits gave the Arrhenius parameters in Table 2.

crease in the exchange rate constant from 4000 to $14000 \mathrm{~s}^{-1}$ at $\mathrm{pH} 7$ and $309 \mathrm{~K}^{[33]}$

\section{Conclusions}

The exchange rate constants of the $\mathrm{H}^{\mathrm{N} \varepsilon}$ protons in the side chains of the four arginine residues and the $\mathrm{H}^{\mathrm{NG}}$ protons in six of the seven lysine residues in human ubiquitin have been determined as a function of temperature over a range between 275 and $305 \mathrm{~K}$. The exchange rate constants vary between about 25 and $2000 \mathrm{~s}^{-1}$ for the $\mathrm{H}^{\mathrm{N \varepsilon}}$ protons in arginines, and between about 700 and $40000 \mathrm{~s}^{-1}$ for the $\mathrm{H}^{\mathrm{N \zeta}}$ protons in lysines. The activation energies in the Arrhenius sense were found to lie in the range $27-75 \mathrm{~kJ} \mathrm{~mol}^{-1}$. The ability to measure such parameters at a physiologically relevant $\mathrm{pH}$ should allow one to obtain deeper insight into interactions between proteins (folded or unfolded) and to map out the contact interfaces in complexes made up of proteins and other macromolecules.

Table 1. Exchange rate constants of the $\mathrm{H}^{\mathrm{N \varepsilon}}$ protons of the arginine and of the terminal $\mathrm{H}^{\mathrm{N} \xi}$ protons of the lysine residues in human ubiquitin. ${ }^{[a]}$

\begin{tabular}{|c|c|c|c|c|c|}
\hline$T[\mathrm{~K}]$ & 275 & 280 & 290 & 300 & 305 \\
\hline$k_{\mathrm{R} 42}\left[\mathrm{~s}^{-1}\right]$ & [b] & $112 \pm 35$ & $455 \pm 138$ & $540 \pm 135$ & $1250 \pm 152$ \\
\hline$k_{\mathrm{R} 54}\left[\mathrm{~s}^{-1}\right]$ & $24 \pm 2$ & $46 \pm 3$ & $117 \pm 5$ & $312 \pm 18$ & $490 \pm 35$ \\
\hline$k_{\mathrm{R} 72}\left[\mathrm{~s}^{-1}\right]$ & $66 \pm 6$ & $114 \pm 7$ & $387 \pm 29$ & $1005 \pm 47$ & $1710 \pm 52$ \\
\hline$k_{\mathrm{R} 74}\left[\mathrm{~s}^{-1}\right]$ & $78 \pm 1$ & $136 \pm 2$ & $409 \pm 10$ & $1188 \pm 18$ & $1856 \pm 21$ \\
\hline$k_{\mathrm{K} 6}\left[10^{3} \mathrm{~s}^{-1}\right]$ & $6.63 \pm 0.69$ & $9.39 \pm 0.64$ & $15.13 \pm 1.49$ & $35.19 \pm 3.90$ & $39.93 \pm 3.90$ \\
\hline$k_{\mathrm{K} 11}\left[10^{3} \mathrm{~s}^{-1}\right]$ & $1.08 \pm 0.24$ & $1.46 \pm 0.19$ & $3.91 \pm 0.31$ & $11.14 \pm 0.59$ & $16.24 \pm 0.90$ \\
\hline$k_{\mathrm{K} 29}\left[10^{3} \mathrm{~s}^{-1}\right]$ & [b] & [b] & $0.41^{[c]}$ & $4.75 \pm 1.24$ & $7.77 \pm 2.12$ \\
\hline$k_{\mathrm{K} 33}\left[10^{3} \mathrm{~s}^{-1}\right]$ & $0.70 \pm 0.23$ & $1.75 \pm 0.24$ & $3.69 \pm 0.32$ & $8.22 \pm 0.50$ & $15.80 \pm 1.10$ \\
\hline$k_{\mathrm{K} 48}\left[10^{3} \mathrm{~s}^{-1}\right]$ & $1.20 \pm 0.11$ & $1.59 \pm 0.10$ & $4.87 \pm 0.29$ & $11.53 \pm 0.49$ & $17.63 \pm 0.83$ \\
\hline$k_{\mathrm{K} 63}\left[10^{3} \mathrm{~s}^{-1}\right]$ & $0.68 \pm 0.14$ & $1.31 \pm 0.13$ & $3.53 \pm 0.21$ & $9.50 \pm 0.36$ & $14.82 \pm 0.59$ \\
\hline
\end{tabular}


Table 2. Activation energies $\left(E_{\mathrm{a}}\right)$ and the pre-exponential factors $(A)$ [see Eq. (6)] of the $\mathrm{H}^{\mathrm{N \varepsilon}}$ protons of the four arginine and the $\mathrm{H}^{\mathrm{N \varepsilon}}$ protons of six lysine residues in human ubiquitin at $\mathrm{pH} 7.45$.

\begin{tabular}{|lll|} 
Residue & $\ln (A)$ & $E_{\mathrm{a}}[\mathrm{kJ} \mathrm{mol}$ \\
\hline R42 & & $64.3 \pm 8.6$ \\
R54 & $32.5 \pm 3.5$ & $68.1 \pm 1.9$ \\
R72 & $33.0 \pm 0.8$ & $75.6 \pm 1.5$ \\
R74 & $37.2 \pm 0.6$ & $73.5 \pm 0.4$ \\
K6 & $36.5 \pm 0.2$ & $27.1 \pm 0.9$ \\
K11 & $41.8 \pm 2.1$ & $36.2 \pm 1.0$ \\
K29 & $67.1 \pm 2.4$ & $40.2 \pm 17.6$ \\
K33 & $79.0 \pm 44.3$ & $34.5 \pm 1.1$ \\
K48 & $63.3 \pm 2.8$ & $35.4 \pm 0.6$ \\
K63 & $64.8 \pm 1.4$ & $36.9 \pm 0.7$ \\
\hline
\end{tabular}
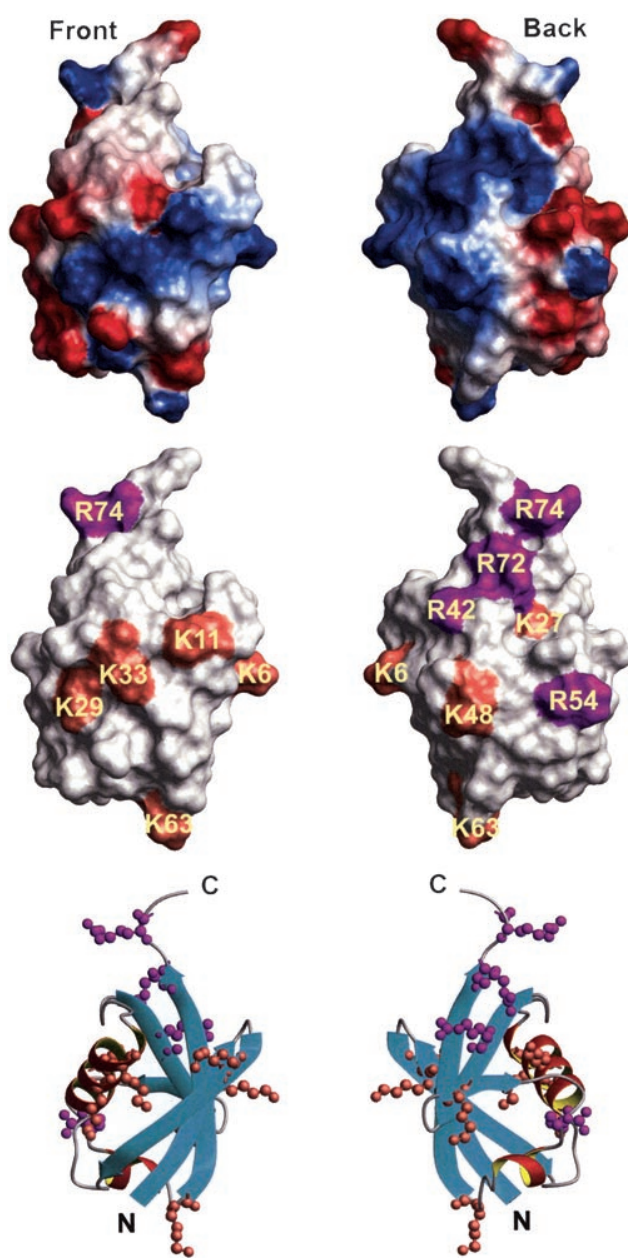

Figure 5. Different representations of human ubiquitin. Top: Electrostatic surface potential plots showing positively charged (blue) and negatively charged (red) regions. Middle: Surface plots showing the seven lysine (orange) and four arginine (magenta) residues, all of which are exposed to the solvent. Bottom: Ribbon diagrams with the side chains of the lysine (orange) and arginine (magenta) residues. Molmol ${ }^{[34]}$ software was used to calculate the electrostatic surfaces and to draw the various representations of human ubiquitin (PDB ID: $1 \mathrm{UBQ}^{[35]}$ ).

\section{Experimental Section}

The proton-exchange rate constants of arginine and lysine side chains were measured in a sample of $\mathrm{U}-\left[{ }^{13} \mathrm{C},{ }^{15} \mathrm{~N}\right]$-labeled human ubiquitin $(0.8 \mathrm{~mm})$, obtained from VLI Research, Inc., at pH 7.45 (at $294 \mathrm{~K})$ in a phosphate buffer $(50 \mathrm{~mm})$. The experiments were carried out on a Bruker Avance spectrometer at $14 \mathrm{~T}\left(600 \mathrm{MHz}\right.$ for $\left.{ }^{1} \mathrm{H}\right)$ fitted with a triple-channel indirect detection probe with three orthogonal gradient coils at temperatures ranging from $275 \mathrm{~K}$ to $305 \mathrm{~K}$. At each temperature, the experiments were repeated three to five times with different numbers of $\pi$-pulses in the CPMG pulse trains ( $n=2$ to 16$)$ and different pulse intervals ( $2 \tau=1.75$ to $10.6 \mathrm{~ms}$ ). The total experimental time for one pair of 2D spectra (A and $\mathrm{B}$ ) varied between $3 \mathrm{~h}$ (at high temperatures) and $6 \mathrm{~h}$ (at low temperatures). At $275 \mathrm{~K}$, the rotational correlation time of ubiquitin is about $9 \mathrm{~ns}$, which corresponds to a protein of about $17 \mathrm{kDa}$ at room temperature. The experimental time could be reduced (or the accessible size of the protein could be increased) by using cryogenically cooled probes. The assignments were taken from Cornilescu et al. ${ }^{[37]}$ The peak intensities were determined by use of the nmrPipe/nlinLS package. ${ }^{[38]}$ From the $A / B$ ratios between the signal intensities in experiments $A$ (without proton decoupling) and $B$ (with proton decoupling) the exchange rate constants were fitted to Equation (1) with the aid of a home-written Mathematica program. The scalar coupling constants $\left({ }^{1} J_{\mathrm{N}, \mathrm{H}}\right)$ measured in a sample of ubiquitin at pH 4.5 were: $93.5 \mathrm{~Hz}$ (R42, R54, R72), 93.9 Hz (R74), and $74 \mathrm{~Hz}$ for the lysine residues. A small correction was applied to account for the presence of $10 \% \mathrm{HDO}$ in the solvent. ${ }^{[26]}$

\section{Acknowledgements}

The authors are indebted to the Centre National de la Recherche Scientifique (CNRS, France), the Agence Nationale pour la Recherche (ANR, France), the Integrated Infrastructure Initiative (I3) in the 6th Framework Program of the EC (Contract RII3-026145, EU-NMR), the Fonds National de la Recherche Scientifique (FNRS, Switzerland), the Commission pour la Technologie et I'Innovation (CTI, Switzerland), and the Laurence Berkeley National Laboratory (LBNL, USA).

Keywords: hydrogen transfer - kinetics - protein structures . proton transfer $\cdot$ scalar relaxation

[1] Y. W. Bai, Chem. Rev. 2006, 106, 1757-1768.

[2] I. M. Russu, Methods Enzymol. 2004, 379, 152-175.

[3] C. E. Dempsey, Prog. Nucl. Magn. Reson. Spectrosc. 2001, 39, 135-170.

[4] M. Guéron, M. Kochoyan, J.-L. Leroy, Nature 1987, 328, 89-92.

[5] A. Krueger, E. Protozanova, M. D. Frank-Kamenetskii, Biophys. J. 2006, 90, 3091-3099.

[6] S. W. Englander, T. R. Sosnick, J. J. Englander, L. Mayne, Curr. Opin. Struct. Biol. 1996, 6, 18-23.

[7] S. Mori, M. O. Johnson, J. M. Berg, P. C. M. van Zijl, J. Am. Chem. Soc. 1994, 116, 11982-11984.

[8] T. L. Hwang, S. Mori, A. J. Shaka, P. C. M. van Zijl, J. Am. Chem. Soc. 1997, $119,6203-6204$.

[9] C. T. W. Moonen, P. Van Gelderen, G. W. Vuister, P. C. M. Van Zijl, J. Magn. Reson. 1992, 97, 419-425.

[10] S. Waelder, L. Lee, A. G. Redfield, J. Am. Chem. Soc. 1975, 97, 2927-2928.

[11] S. Mori, P. C. M. van Zijl, D. Shortle, Proteins Struct. Funct. Genet. 1997, 28, 325-332.

[12] Y. Bai, J. S. Milne, L. Mayne, S. W. Englander, J. Protein Chem. 1993, 17, 75-86. 
[13] P. C. M. van Zijl, C. K. Jones, J. Ren, C. R. Malloy, A. D. Sherry, Proc. Natl. Acad. Sci. USA 2007, 104, 4359-4364.

[14] J. Y. Zhou, P. C. M. van Zijl, Progr. Nucl. Magn. Reson. Spectrosc. 2006, 48, 109-136.

[15] A. A. Gilad, M. T. McMahon, P. Walczak, P. T. Winnard, V. Raman, H. W. M. van Laarhoven, C. M. Skoglund, J. W. M. Bulte, P. C. M. van Zijl, Nat. Biotechnol. 2007, 25, 217-219.

[16] K. M. Ward, A. H. Aletras, R. S. Balaban, J. Magn. Reson. 2000, 143, 79-87.

[17] J. Pfeuffer, W. Dreher, E. Sykova, D. Leibfritz, Magn. Reson. Imaging 1998, 16, 1023-1032.

[18] J. Iwahara, Y. S. Jung, G. M. Clore, J. Am. Chem. Soc. 2007, 129, 29712980.

[19] N. R. Skrynnikov, R. R. Ernst, J. Magn. Reson. 1999, 137, 276-280.

[20] G. Gemmecker, W. Jahnke, H. Kessler, J. Am. Chem. Soc. 1993, 115, $11620-11621$.

[21] H. S. Gutowsky, D. W. McCall, C. P. Slichter, J. Chem. Phys. 1953, 21, 279292.

[22] F. Blomberg, W. Maurer, H. Rüterjans, Proc. Natl. Acad. Sci. USA 1976, 73 1409-1413.

[23] P. Macheroux, S. Ghisla, C. Sanner, H. Rüterjans, F. Müller, BMC Biochem. 2005, 6:26.

[24] S. Meiboom, D. Gill, Rev. Sci. Instrum. 1958, 29, 688-691.

[25] H. Y. Carr, E. M. Purcell, Phys. Rev. 1954, 94, 630-638.
[26] F. Kateb, P. Pelupessy, G. Bodenhausen, J. Magn. Reson. 2007, 184, 108113.

[27] H. Geen, R. Freeman, J. Magn. Reson. 1991, 93, 93-141.

[28] L. Emsley, G. Bodenhausen, J. Magn. Reson. 1992, 97, 135-148.

[29] L. Emsley, G. Bodenhausen, Chem. Phys. Lett. 1990, 168, 297-303.

[30] A. J. Shaka, J. Keeler, T. Frenkiel, R. Freeman, J. Magn. Reson. 1983, 52, 335-338.

[31] A. L. Davis, J. Keeler, E. D. Laue, D. Moskau, J. Magn. Reson. 1992, 98 207-216.

[32] G. A. Morris, R. Freeman, J. Am. Chem. Soc. 1979, 101, 760-762.

[33] E. Liepinsh, G. Otting, Magn. Reson. Med. 1996, 35, 30-42.

[34] R. Koradi, M. Billeter, K. Wüthrich, J. Molec. Graphics 1996, 14, 51-55.

[35] S. Vijaykumar, C. E. Bugg, W. J. Cook, J. Mol. Biol. 1987, 194, 531-544.

[36] M. Sundd, A. D. Robertson, J. Mol. Biol. 2003, 332, 927-936.

[37] G. Cornilescu, J. L. Marquardt, M. Ottiger, A. Bax, J. Am. Chem. Soc. 1998, 120, 6836-6837.

[38] F. Delaglio, S. Grzesiek, G. W. Vuister, G. Zhu, J. Pfeifer, A. Bax, J. Biomol. NMR 1995, 6, 277-293.

Received: October 8, 2007

Published online on February 5, 2008 